\title{
Misfit dislocations in nanoscale ferroelectric heterostructures
}

\author{
V. Nagarajan, C. L. Jia, H. Kohlstedt, and R. Waser \\ Institut für Festkörperforschung (IFF), Forschungzentrum Jülich D 52425, Germany \\ I. B. Misirlioglu and S. P. Alpay ${ }^{\text {a) }}$ \\ Department of Materials Science and Engineering, Institute of Materials Science, \\ University of Connecticut, Storrs, Connecticut 06269 \\ R. Ramesh \\ Department of Materials Science and Engineering and Department of Physics, University of California, \\ Berkeley, California 94720
}

(Received 8 February 2005; accepted 17 March 2005; published online 6 May 2005)

\begin{abstract}
We present a quantitative study of the thickness dependence of the polarization and piezoelectric properties in epitaxial (001) $\mathrm{PbZr}_{0.52} \mathrm{Ti}_{0.48} \mathrm{O}_{3}$ films grown on (001) $\mathrm{SrRuO}_{3}$-buffered (001) $\mathrm{SrTiO}_{3}$ substrates. High-resolution transmission electron microscopy reveals that even the thinnest films $(\sim 8 \mathrm{~nm})$ are fully relaxed with a dislocation density close to $10^{12} \mathrm{~cm}^{-2}$ and a spacing of approximately $12 \mathrm{~nm}$. Quantitative piezoelectric and ferroelectric measurements show a drastic degradation in the out-of-plane piezoelectric constant $\left(d_{33}\right)$ and the switched polarization $(\Delta P)$ as a function of decreasing thickness. In contrast, lattice-matched ultrathin $\mathrm{PbZr}_{0.2} \mathrm{Ti}_{0.8} \mathrm{O}_{3}$ films that have a very low dislocation density show superior ferroelectric properties. Supporting theoretical calculations show that the variations in the strain field around the core of the dislocation leads to highly localized polarization gradients and hence strong depolarizing fields, which result in suppression of ferroelectricity in the vicinity of a dislocation. (c) 2005 American Institute of Physics.
\end{abstract}

[DOI: $10.1063 / 1.1922579]$

Understanding nanoscale ferroelectrics has become a topic of intense fundamental research. ${ }^{1,2}$ Recent studies show that lattice engineering and internal stresses have a major role in the structural and physical properties of ultrathin ferroelectric films. ${ }^{3-5}$ In lattice-matched (or epitaxial) films and heterostructures, the strain due to lattice mismatch between the film and the substrate can be relaxed during film growth. by the formation of orthogonal arrays of misfit dislocations at the film-substrate interface. ${ }^{6-11}$ For epitaxial 10 nm high $\mathrm{PbZr}_{0.52} \mathrm{Ti}_{0.48} \mathrm{O}_{3}$ [PZT (52/48)] nanoislands on $\mathrm{Nb}$ doped $\mathrm{SrTiO}_{3}$ (STO), the strain field associated with the dislocation core was found to be propagating into the ferroelectric layer with a height of $\sim 4 \mathrm{~nm}$ and a width of $\sim 8 \mathrm{~nm}$, a significant volume fraction of the islands. ${ }^{4}$

Recent theoretical results ${ }^{12}$ for an epitaxial PTO thin film with periodic misfit dislocations predict that the strong coupling of the dislocation strain field with the polarization leads to high local polarization gradients. This results in strong depolarizing fields around a dislocation in a region of 5-10 $\mathrm{nm}$ in diameter around the core, that ultimately suppress ferroelectricity. ${ }^{13,14}$ Misfit dislocations should thus act as an extrinsic dominating factor in the scaling of polarization and all physical properties as a function of thickness. In this letter, we provide a systematic quantitative study on the role of dislocations on the stability ferroelectricity in epitaxial ferroelectric thin films.

Epitaxial (001) PZT (52/48) films were grown on (001) $\mathrm{SrRuO}_{3}$ (SRO)-buffered (001) $\mathrm{SrTiO}_{3}$ (STO) substrates. The thickness of the PZT (52/48) layer was varied from $200 \mathrm{~nm}$ down to $12 \mathrm{~nm}$ thickness. The details of the growth method and parameters are given elsewhere. ${ }^{15}$ In order to compare

a) Author to whom correspondence should be addressed; electronic mail: p.alpay@ims.uconn.edu and contrast the effect of misfit dislocations, very well lattice-matched PZT (20/80)/SRO/STO heterostructures were also prepared from $80 \mathrm{~nm}$ down to $4 \mathrm{~nm}$. In order to avoid complications from $90^{\circ}$ domain formation, ${ }^{16}$ we chose $80 \mathrm{~nm}$ as the upper bound for the PZT (20/80) films.

$\mathrm{X}$-ray synchrotron measurements confirmed that the SRO layer was pseudomorphic with the STO substrate with an in-plane lattice parameter of $0.3905 \mathrm{~nm}$ at room temperature (RT) and $0.3930 \mathrm{~nm}$ at the growth temperature $T_{G}$ $=600{ }^{\circ} \mathrm{C}$. Additional $\mathrm{x}$-ray and high-resolution transmission electron microscopy (TEM) diffraction verified the epitaxial orientational relationship: (001)PZT[100]PZT |(001)SRO [100]STO\|(001)STO[100]STO. X-ray diffraction reciprocal space mapping (not shown) of the PZT layers confirmed them to be tetragonal. At $T_{G}$, the lattice parameter of PZT $(52 / 48)$ is $0.4084 \mathrm{~nm}$; therefore, the misfit between SRO and PZT $(52 / 48)$ at $T_{G}$ is $-3.93 \%$. Employing the MatthewsBlakeslee criteria ${ }^{6}$ and using the elastic moduli of PZT (52/48), ${ }^{17}$ the critical thickness for misfit dislocation formation was calculated to be $\sim 2.3 \mathrm{~nm}$ and the nominal dislocation spacing to be $12.8 \mathrm{~nm}$ for the $12 \mathrm{~nm}$ and $10.5 \mathrm{~nm}$ for the $160 \mathrm{~nm}$ thick film, respectively. In contrast, the in-plane lattice parameter for PZT $(20 / 80)$ at $T_{G}$ is $0.396 \mathrm{~nm}$; thus, the lattice mismatch at growth temperature is only around $-0.7 \%$ resulting in a theoretical $h_{c} \sim 10 \mathrm{~nm}$ and a spacing of about $30 \mathrm{~nm}$.

Figure 1(a) is a representative cross-sectional, TEM image of a $20 \mathrm{~nm}$ thick PZT (52/48) film on SRO/STO. The ferroelectric layer is rough even at the nanoscale and has a large density of dislocations indicated by the strain contrast in the image. In comparison, Fig. 1(b) is a low-magnification image of a $20 \mathrm{~nm}$ thick PZT (20/80) film. The dashed lines in Fig. 1(b) indicate the interfaces between the PZT, SRO, and STO layers, respectively. The interfaces are smoother 


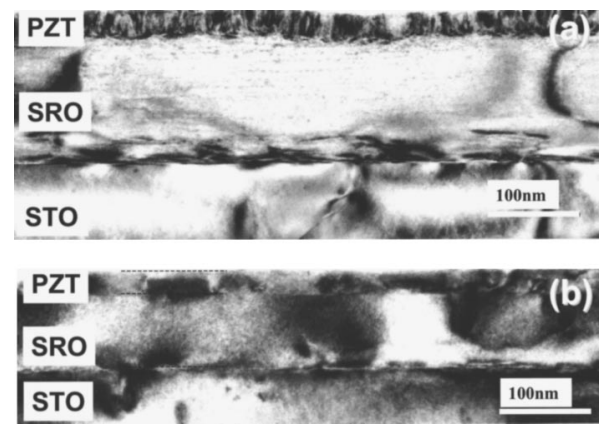

FIG. 1. Comparison of TEM images for PZT (52/48) (top) and PZT (20/80) (bottom) films, both $20 \mathrm{~nm}$ thick. The image shows that PZT $(52 / 48)$ has a large density of dislocations and rough interfaces due to lattice mismatch. PZT (20/80) film shows a relatively smooth and sharp interface as a result of the closer lattice matching.

with a significantly reduced strain contrast and the density of defects is greatly reduced. Figure 2(a) shows a highresolution image of an $8 \mathrm{~nm}$ thick PZT (52/48) sandwiched between the top and bottom SRO layers. An array of misfit dislocations is observed at the interface between the PZT $(52 / 48)$ and the bottom SRO layer as indicated by open arrows. The majority of the dislocations are located in the SRO layer away from the interface plane. Some of the dislocations dissociate into two partials to lower the energy associated with the lattice strain. Figure 2(b) shows a magnified region around the dislocation core within the white frame. Dissociation of the dislocation can be clearly seen as indicated by two arrows. The corresponding Burger's vectors for the two dissociating edge-type partials are $a / 2$ [101] and $a / 2$ [101] leading to a sum Burger's vector $\mathbf{b}=a[100]$.

Lattice strain in the vicinity of the dislocation was studied by studying the changes in lattice fringe spacings using geometric phase analysis of the lattice fringe image. ${ }^{18}$ In calculating the lattice spacing variations in the framed area

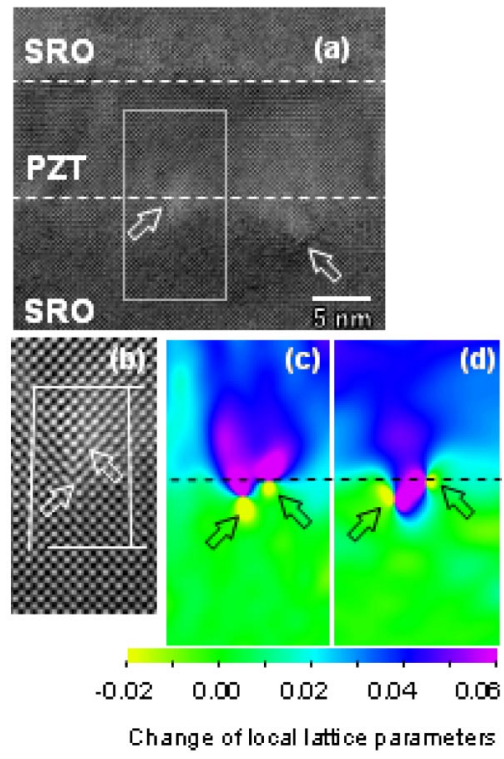

FIG. 2. (Color online) (a) HRTEM image of a $8 \mathrm{~nm} \mathrm{SRO/PZT(52/48)/SRO}$ heterostructure. The arrows point toward misfit dislocations. The dislocation has split into two partials to further reduce the energy associated with the lattice strain. (b) A magnified region around the dislocation core within the white frame. Dissociation of the dislocation into two partials can be clearly seen as indicated by two arrows. (c) and (d) show maps of spacing changes along the in-plane axis [100] and out-of-plane axis [001], respectively.
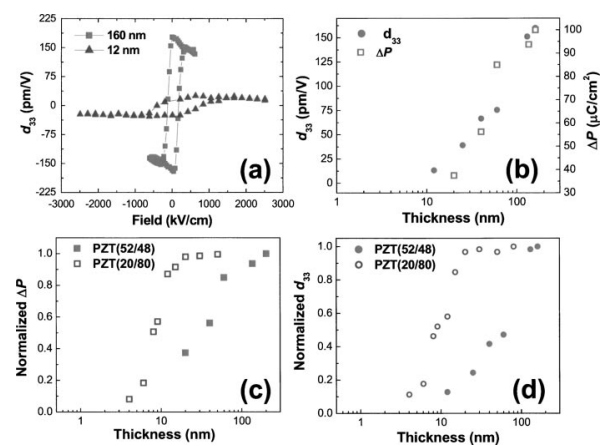

FIG. 3. Piezoelectric and polarization characterization of the PZT (52/48) and PZT (20/80) thickness series: (a) Piezoelectric hysteresis loops for the $12 \mathrm{~nm}$ (triangles) and $160 \mathrm{~nm}$ (squares) PZT (52/48) films. (b) Measured out-of-plane piezoelectric constant $d_{33}$ and switchable polarization $(\Delta P)$ for PZT $(52 / 48)$ as a function of film thickness. (c) Out-of-plane $d_{33}$ and (d) switchable polarization normalized with respect to their individual "unsuppressed" values as a function of film thickness. In the case of the PZT (52/48) films, a steep drop begins at $\sim 100 \mathrm{~nm}$, a thickness much greater than the case of the PZT (20/80) film $(\sim 12 \mathrm{~nm})$.

of Fig. 2(b), the SRO lattice fringes in the bottom part of the frame were used as a spacing reference. Figures 2(c) and 2(d) show the maps of spacing changes along the in-plane axis [100] and out-of-plane axis [001], respectively. The scale in Figs. 2(c) and 2(d) quantifies the changes in the local lattice parameter with respect to the SRO fringes. It can be seen that the SRO layer is locally compressed while the PZT layer has significant in-plane tensile strain close to the core. The extra half-plane is in the SRO layer with the core penetrating into this layer away from the interface to minimize excessive elastic energy associated with the core. This is frequently observed in films on substrates where the film is elastically stiffer than the substrate. ${ }^{19}$ The abrupt changes of lattice stain, from compressive to tensile across the edge dislocation cores, are well characterized by variations in the color scale. Above the dislocation core, a tensile lattice strain is clearly seen in the two maps in comparison with the areas away from the dislocation cores and parallel to the interface. The lattice expansion in-plane is stronger than the one outof-plane and extends far into the PZT (52/48) layer.

Quantitative piezoresponse force microscopy was used to extract the out-of-plane piezoelectric coefficient $d_{33}$. To ensure a uniform electric-field distribution, the measurements were made using a top electrode and a conductive Pt-Ir tip. The displacement of the cantilever was calibrated using $x$-cut quartz, which has a known piezoelectric coefficient $(2.3 \mathrm{pm} / \mathrm{V})$. Figure $3(\mathrm{a})$ shows $d_{33}$ loops for two PZT (52/48) thicknesses: $12 \mathrm{~nm}$ (triangles) and $160 \mathrm{~nm}$ (squares) thick films. The plot shows a dramatic reduction in the piezoelectric coefficient for the $12 \mathrm{~nm}$ film compared to the $160 \mathrm{~nm}$ thick film. For this composition, $d_{33}$ for a clamped (001)-oriented epitaxial film without any suppression or extrinsic effects, is expected to be $\sim 157 \mathrm{pm} / \mathrm{V}$ (Ref. 20) as shown by the $160 \mathrm{~nm}$ thick film. This drops significantly for the $12 \mathrm{~nm}$ film, which shows average remanent $d_{33}$ of only $30 \mathrm{pm} / \mathrm{V}$. In Fig. 3(b), we plot $d_{33}$ at remanance as a function of film thickness. It shows a systematic and sharp fall when the thickness is below $100 \mathrm{~nm}$, indicating the detrimental effect of misfit dislocations. We also measured the switchable polarization $(\Delta P)$, which is the difference between the switched $\left(P^{*}\right)$ and nonswitched $\left(P^{\hat{}}\right)$ responses. ${ }^{21}$ We used this instead of $2 \cdot P_{R}$, where $P_{R}$ is the remanent polarization, o AlP license or copyright, see http://apl.aip.org/apl/copyright.jsp 

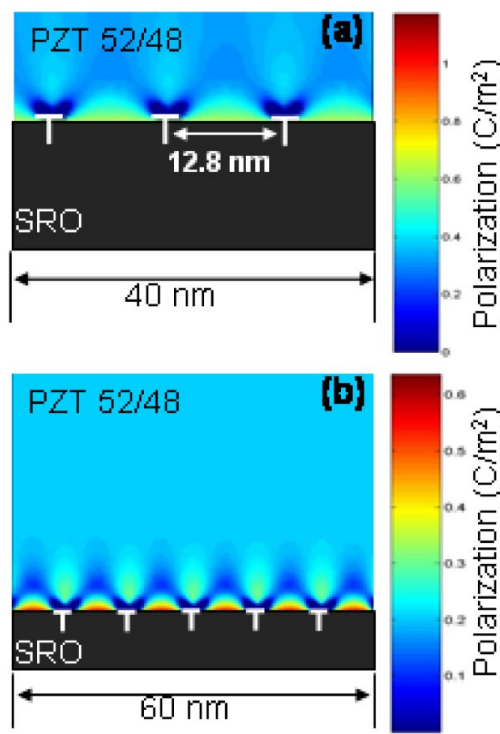

FIG. 4. (Color online) The polarization distribution around periodic misfit dislocations with $\mathbf{b}=a[\overline{100}]$ in (a) $12 \mathrm{~nm}$ and (b) $200 \mathrm{~nm}$ thick (001) PZT (52/48) film on (001) SRO-buffered (001) STO substrate, assuming a deposition temperature of $600{ }^{\circ} \mathrm{C}$. Film areas shown in (a) and (b) represent $12 \mathrm{~nm} \times 40 \mathrm{~nm}$ and $30 \mathrm{~nm} \times 60 \mathrm{~nm}$ cross section across the film thickness in the $x z$-plane, respectively. Individual elements in the simulations were $\sim 0.4 \mathrm{~nm}$.

as it is less likely to be convoluted by leakage. We find that similar to $d_{33}$, the $\Delta P$ also drops drastically for thicknesses below $100 \mathrm{~nm}$. This drop begins at a thickness much greater than that predicted for fully commensurate ferroelectric heterostructures by theory ${ }^{22,23}$ or experiments. ${ }^{24}$ It, therefore, clearly illustrates the dominant extrinsic role dislocations play in the size effects of nanoscale ferroelectrics. This is evident in Figs. 3(c) and 3(d), where we compare $d_{33}$ and $\Delta P$ values of the PZT (20/80) versus PZT (52/48). For the sake of comparing both compositions on one scale, the values have been normalized to the unsuppressed theoretical values. The lattice-matched PZT (20/80) system shows no scaling in $d_{33}$ or $\Delta P$ down to $15 \mathrm{~nm} .{ }^{24}$ In comparison, the drop in the PZT (52/48) system is much sooner; thereby exhibiting the overriding role of dislocations in the size effects of ferroelectrics.

To probe this further, we carried out a thermodynamic analysis to investigate the role of dislocations in ferroelectric materials using a methodology based on a modified meanfield Landau-Devonshire formalism incorporating the elastic energy of the dislocations, its electromechanical coupling to the polarization, and the internal field due to polarization gradients given by Maxwell's relations. The details of this method are given elsewhere. ${ }^{12}$ We analyzed the polarization distribution around misfit dislocations with $\mathbf{b}=a[\overline{1} 00]$ in (001) PZT (52/48) films with 12 and $200 \mathrm{~nm}$ thickness on thick (001) STO substrates with fully relaxed SRO buffer layers (SRO/STO). The internal stresses due to lattice mismatch were coupled with the stress field of dislocations in the linear elastic limit. As shown in Figs. 4(a) and 4(b), due to strong strain gradients, there is a drastic variation in the polarization near the film-substrate interface that should result in the formation of $\sim 10$ and $\sim 15 \mathrm{~nm}$ thick ferroelectrically dead layers in 12 and $200 \mathrm{~nm}$ films, respectively. These plots also display the thickness dependence of the dead layer-to-film thickness ratio. As films get thicker, the equilibrium dislocation density increases and hence the dislocation periodicity decreases resulting in better relaxation of epitaxial stresses. The thickness of the dead layer depends on the dislocation density, which theoretically levels off above $\sim 100 \mathrm{~nm}$ in PZT (52/48) films on SRO/STO.

These results have several implications in terms of properties of ultrathin ferroelectric films and nanostructures. Obviously, there exists a significant volume that will not contribute to the polarization, dielectric, piezoelectric, and pyroelectric response. Furthermore, because of this dead layer, the applied electrical field that is necessary to activate the unique properties of ferroelectrics will be screened. These regions also serve as pinning centers for reversible $180^{\circ}$ and non- $180^{\circ}$ domain-wall motion in the presence of an applied field, and thereby reduce the extrinsic contribution to all physical properties. The detrimental effect of such regions will be enhanced in nanoscale ferroelectric films and heterostructures, and hence play a critical extrinsic role in size effect studies of ferroelectrics.

This work was supported by the National Science Foundation (NSF) under Grant Nos. DMR-0132918, NSFMRSEC DMR-0080008, and (an NSF US-Europe program) DMR-0244288. One of the authors (V. N.) also acknowledges the support of the Alexander von Humboldt Foundation for his stay in Germany.

${ }^{1}$ D. D. Fong, G. B. Stephenson, S. K. Streiffer, J. A. Eastman, O. Auciello, P. H. Fuoss, and C. Thompson, Science 304, 1650 (2004).

${ }^{2}$ I. I. Naumov, L. Bellaiche, and H. Fu, Nature (London) 432, 737 (2004). ${ }^{3}$ K. J. Choi, M. Biegalski, Y. L. Li, A. Sharan, J. Schubert, R. Uecker, P. Reiche, Y. B. Chen, X. Q. Pan, V. Gopalan, L. Q. Chen, D. G. Schlom, and C. B. Eom, Science 306, 1005 (2004).

${ }^{4}$ M. W. Chu, I. Szafraniak, R. Scholz, C. Harnagea, D. Hesse, M. Alexe, and U. Gosele, Nat. Mater. 3, 87 (2004).

${ }^{5}$ S. P. Alpay, I. B. Misirlioglu, A. Sharma, and Z.-G. Ban, J. Appl. Phys. 95, 8118 (2004)

${ }^{6}$ J. W. Matthews and A. E. Blakeslee, J. Cryst. Growth 27, 118 (1974).

${ }^{7}$ S. Stemmer, S. K. Streiffer, F. Ernst, and M. Ruhle, Phys. Status Solidi A 147, 135 (1995)

${ }^{8}$ S. Y. Hu, Y. L. Li, and L. Q. Chen, J. Appl. Phys. 94, 2542 (2003).

${ }^{9}$ L. Lymperakis, J. Neugebauer, M. Albrecht, T. Remmele, and H. P. Strunk, Phys. Rev. Lett. 93196401 (2004).

${ }^{10}$ G. F. Neumark, Phys. Rev. Lett. 21, 1252 (1968).

${ }^{11}$ C. L. Canedy, H. Li, S. P. Alpay, L. Salamanca-Riba, A. L. Roytburd, and R. Ramesh, Appl. Phys. Lett. 77, 1695 (2000).

${ }^{12}$ S. P. Alpay, I. B. Misirlioglu, V. Nagarajan, and R. Ramesh, Appl. Phys. Lett. 85, 2044 (2004).

${ }^{13}$ A. M. Bratkovsky and A. P. Levanyuk, Phys. Rev. B 63, 132103 (2001).

${ }^{14}$ A. M. Bratkovsky and A. P. Levanyuk, Phys. Rev. B 66, 184109 (2002).

${ }^{15}$ C. L. Jia, J. R. Contreras, U. Poppe, H. Kohlstedt, R. Waser, and K. Urban, J. Appl. Phys. 92, 101 (2002).

${ }^{16}$ S. P. Alpay and A. L. Roytburd, J. Appl. Phys. 83, 4714 (1998).

${ }^{17}$ Landolt-Börnstein, Numerical Data and Functional Relationships in Science and Technology (Springer, Berlin, 1982).

${ }^{18}$ M. J. Hytch, E. Snoeck, and R. Kilaas, Ultramicroscopy 74, 131 (1998).

${ }^{19}$ X. Wu and G. C. Weatherly, Semicond. Sci. Technol. 18, 307 (2003).

${ }^{20}$ L. Chen, V. Nagarajan, R. Ramesh, and A. L. Roytburd, J. Appl. Phys. 94, 5147 (2003).

${ }^{21}$ P. K. Larsen, G. L. M. Kampschoer, M. J. E. Ulenaers, G. A. C. M. Spierings, and R. Cuppens, Appl. Phys. Lett. 59, 611 (1991).

${ }^{22}$ J. Junquera and P. Ghosez, Nature (London) 422, 506 (2003).

${ }^{23}$ A. G. Zembilgotov, N. A. Pertsev, H. Kohlstedt, and R. Waser, J. Appl. Phys. 91, 2247 (2002).

${ }^{24}$ V. Nagarajan, S. Prasertchoung, T. Zhao, H. Zheng, J. Ouyang, R. Ramesh, W. Tian, X. Q. Pan, D. M. Kim, C. B. Eom, H. Kohlstedt, and R. Waser, Appl. Phys. Lett. 84, 5225 (2004). 